\title{
Detection of Klebsiella pneumoniae DNA by PCR-based CRISPR-IbCas12a system
}

Shang Wang ${ }^{1 *}$, Ying Tang ${ }^{1,2,{ }^{*}}$, Jiehong $\mathrm{Wei}^{3}$, Shan Wang ${ }^{1}$, Guoyu Peng ${ }^{1}$, Yuqing $\mathrm{Li}^{1,3}$, Song $\mathrm{Wu}^{1,3,4, \#}$

${ }^{1}$ Institute of Urology, The Third Affiliated Hospital of Shenzhen University, Shenzhen 518000, China.

${ }^{2}$ Teaching Center of Shenzhen Luohu Hospital, Shantou University Medical College, Shantou 515000, China.

${ }^{3}$ Medical Laboratory of Shenzhen Luohu People's Hospital, Shenzhen, China.

${ }^{4}$ South China Hospital, Health Science Centre, Shenzhen University, Shenzhen, China.

Corresponding author: Song Wu, Ph.D. M.D, wusong@mail.szu.edu.cn;

\#The authors wish it to be known that, in their opinion, the first 2 authors should be regarded as joint First Authors. 


\section{Abstract}

Klebsiella pneumonia (K. pneumoniae) is a Gram-negative bacterium that causes nosocomial infections in the lung, bloodstream, and urinary tract. Therefore, detecting $K$. pneumoniae in early time is important in preventing severe infections. However, clinical detection of $K$. pneumoniae requires a long time of agar plate culture. Nucleic acid detection like qPCR is precise but requires expensive equipment. Recent research reveals that collateral cleavage activity of CRISPR-LbCas12a has been applied in nucleic acid detection. In this study, PCR combined with CRISPR-LbCas12a targeting the $K$. pneumoniae system was established. This system showed excellent detection specificity and sensitivity in both bench work and clinical samples. Due to its advantages, its application can meet different detection requirements in health centers where qPCR is not accessible.

Keywords: CRISPR-Cas, Nucleic acid detection, Klebsiella pneumonia Abbreviations: Polymerase Chain Reaction (PCR), Klebsiella pneumonia (K. pneumoniae), Urinary Tract Infection (UTI), bloodstream infections (BSI) 


\section{Graphical Abstract}

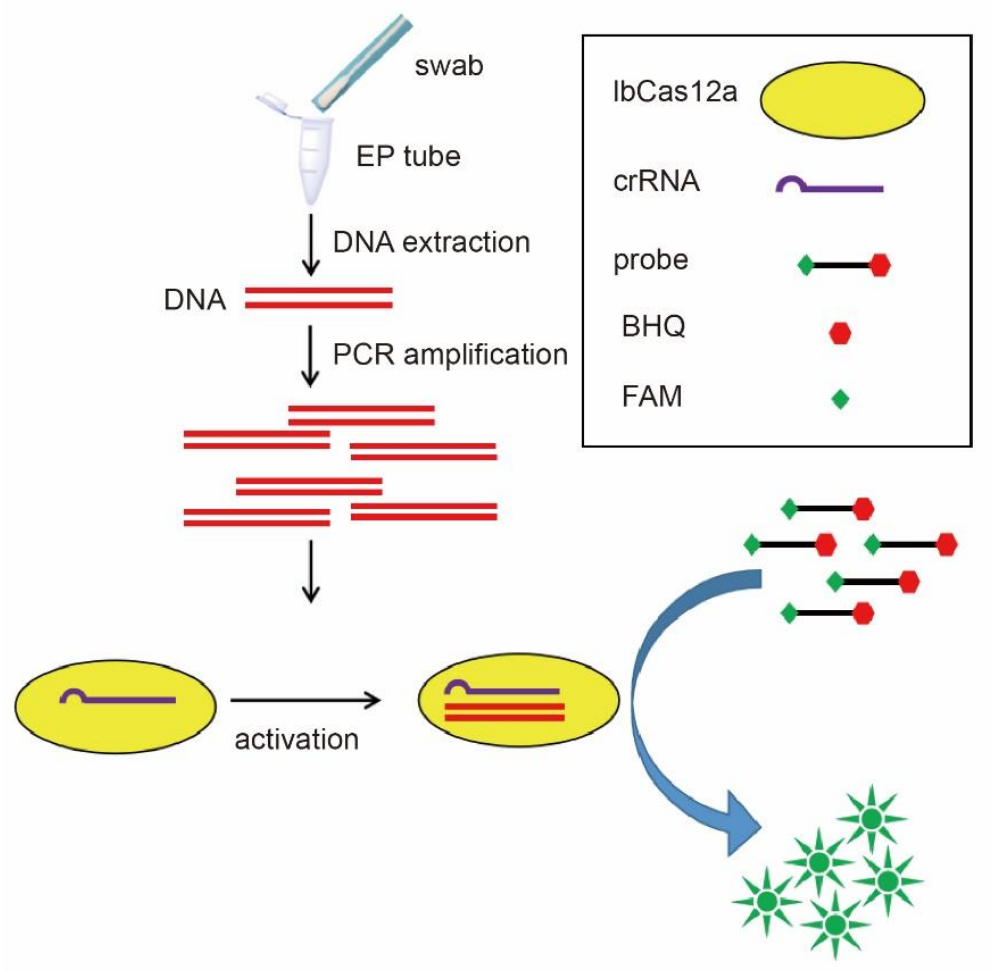




\section{Introduction}

Klebsiella pneumonia (K. pneumoniae) is a family of Gram-negative bacteria that causes nosocomial infections in the bloodstream, wound, urinary tract [1]. Hypervirulent $K$. pneumoniae strains such as $\mathrm{K} 1, \mathrm{~K} 2$, and $\mathrm{K} 5$, have emerged worldwide and caused severe infections, including liver abscess, pneumonia with a mortality rate as high as $20 \%$ 30\% [2]. Additionally, nearly $44.5 \%$ K. pneumoniae strains are multi-drug resistant. [3] Due to the risks of mortality and the difficulty in treating, detecting K. pneumoniae in patients and ventilators is vital in curing and preventing severe infections.

K. pneumoniae colonies derived from clinical samples under 24-48 hours of plate culture present features that could be diagnosed by well-trained personnel [4]. Despite the low cost and the simplicity of operation, plate culture is time-consuming and sometimes produces false-negative results for the colonies that may not be typical $[5,6]$. In contrast, nucleic acid detection methods that detect K. pneumoniae-specific DNA fragments are more advantageous. Quantitative real-time PCR or qPCR is the most used nucleic acid detection method. It can detect targeted pathogens and even identify subtypes, including $\mathrm{K} 1$ and $\mathrm{K} 2$ strains that are multidrug-resistant and hypervirulent [7]. However, qPCR requires expensive equipment, which restricts its application in the healthcare center.

The CRISPR-Cas systems have been discovered to cleavage target DNA or RNA under the guidance of crRNA in a base-pairing manner[8]. Recent research shows that Cas13 and Cas12 exhibit collateral cleavage activity that could degrade probes if crRNA perfectly base-pairs targeted RNAs or DNAs $[9,10]$. Combined with DNA amplification and detection methods, Cas12 and Cas13 
were applied to detect nucleic acid [11]. The detection workflow only requires $37^{\circ} \mathrm{C}$ incubation and does not rely on complicated equipment. To date, to combat COVID-19, lots of nucleic acid detection workflows based on Cas13 and Cas12 have been developed [12,13].

Compared to Cas13 system, Cas12 showed advantages in following aspects:

1, Cas12 requires DNA as the target, but Cas13 requires RNA. Because the technologies to directly amplify RNA are not mature enough, the Cas 13 detection system needs in vitro transcription process.

2, The detecting probes used in Cas12 are DNA, while Cas13's probes are RNAs that are easily degraded to produce the false-positive effect.

3, The crRNA of Cas13 is as large as $64 \mathrm{nt}$, while that of Cas12 could be as short as $35 \mathrm{nt}$.

The RNA length as short as $35 \mathrm{nt}$ could be easily synthesized in the industry[11]. In this study, instead of using crRNA derived from in vitro. transcription, which requires complicated processes in enzyme digestion and RNA purification but produces an uncertain amount of RNA, we used synthetic 35nt RNA as crRNA for its stability in quality control and convenience in quantitation. In addition, the slight progression makes the system more stable and more appliable to prepare premix of reagent for clinical use.

Despite these advantages, the CRISPR-Cas12 detection method targeting K. pneumoniae has not been reported. We established a sensitive nucleic acid detection method based on CRISPRCas12 and PCR to detect K. pneumoniae. It produces authentic results in less than 4 hours and requires less expensive equipment. It may help community healthcare centers to accomplish nucleic acid detection. 


\section{Materials and Methods}

Nucleic Acid preparation

crRNAs were designed to target 16sRNA, YP_005224572.1 and IF-2 gene according to

protocol [9]. RNA nucleotides were chemically synthesized without 5'-phospohrylation by (Transheep, China). crRNA consists of 19nt of common sequences and 17nt for recognizing target [14]. The RNA sequences are as follows:

crRNA-1: 5'-AAU UUC UAC UGU UGU AGA UAC AUC CGA CUU GAC AGA-3'

crRNA-2: 5'-AAU UUC UAC UGU UGU AGA UGA AUG CAG UUC CCA GGU-3'

crRNA-3: 5'-AAU UUC UAC UGU UGU AGA UUC CAC ACU UCC GGA UAG-3'

crRNA-4: 5'-AAU UUC UAC UGU UGU AGA UCC GCU GGU ACC GGC AUC-3'

crRNA-5: 5'-AAU UUC UAC UGU UGU AGA UCG UGC UGG AUA GCU UCG-3'

FAM-BHQ labeled DNA probe was synthesized by Sangon Corp (China).

DNA targets for crRNA testing were synthesized by Sangon Corp (China). The sequences are as follows:

Target-1: 5'-GGT CTG TCA AGT CGG ATG TGA AAT C-3'

Target-2: 5'-TCAACC TGG GAA CTG CAT TCG AAA CTG-3'

Target-3: 5'-GGG CTA TCC GGA AGT GTG GAT AAA CG-3'

Target-4: 5'-GCA GAT GCC GGT ACC AGC GGG AAA AC-3'

Target-5: 5'-TAT CGA AGC TAT CCA GCA CGC TAA AGC-3'

Probe sequence and modification is 5'-FAM-TTTTTT-BHQ-3'. 
Standard DNA carrying K. pneumoniae 16sRNA, YP_005224572.1 and IF-2 gene fragments were synthesized and cloned to pUC-19 vector. Full sequences are as follows:

5 -

AGGAAGGCCGGTGAGGTTAATAACCTCTCGATTGACGTTACCCGCAGAAGAAGCACCGG

CTAACTCCGTGCCAGCAGCCGCGGTAATACGGAGGGTGCAAGCGTTAATCGGAATTACT

GGGCGTAAAGCGCACGCAGGCGGTCTGTCAAGTCGGATGTGAAATCCCCGGGCTCAAC

CTGGGAACTGCATTCGAAACTGGCAGGCTAGAGTCTTGTAGAGGGGGGTAGAATTCCAG

GTGTAGCGGTGAAATGCGTAGAGATCTGGAGGAATACCGGTGGCGAAGGCGGCCCCCT

GGACAAAGACTGACGCTCAGGTGCGAAA (16S RNA, 785-1105)

CGAGGTTTACGTCTCAACCGGCTGGGGATCCACCACGAGCGGCTGCCGCCCGGGCGG

CGCACCTCTTATCCACACGCGGAGAGCGATGAGGAAGAGTTCATCTACGTGCTGGAGGG

CTATCCGGAAGTGTGGATAAACGGCTATCTCTGGAAGCTGGAGCCCGGCGACAGCGTGG

GTTTTCCCGCTGGTACCGGCATCTGCCACACCTTTCTCAATAACACCGAGCAGGAAGTTC

GTCTG (RefSeq: YP_005224572.1, 145-384)

CTGGTGGTGGGCGAGGCCAACAAGAAGTACAACCGCATTTATTACCGGCATGATCACCTT

CCTGGATACCCCGGGCCACGCCGCGTTTACCTCCATGCGTGCTCGTGGCGCGCAGGCG

ACGGATATCGTGGTTCTGGTGGTGGCGGCAGACGACGGCGTGATGCCGCAGACTATCGA

AGCTATCCAGCACGCTAAAGCGGCGCAGGTACCGGTGGTAGTGGCGGTGAACAAGATCG

ATAAGCCAGAAGCCGATCCGGATCGCGTGAAGAACGAACTGTCCCAGTACGGCATCCTG

CCGGAAGAGTGGGGCGGCGAGAGCCAGTTCGTCCACGTTTCCGCGAAAGCGGGTACC

GGCATCGACGAC (IF-2 GenBank: CP052761.1; LOCUS: CP052761; gene ID: inf B 1,329$1,647)-3$ 
DNA extraction and quantification

Clinical samples were swabs of sputa. According to the manufacturer's protocol, swabs were dipped in cell lysate and processed genomic DNA extraction using the DNA extraction Kit (Tianlong science \& technology). Extracted DNA samples were quantified by Nanodrop ${ }^{\complement}$ and preserved in $80^{\circ} \mathrm{C}$ before use.

PCR and qPCR

PCR system was carried in $20 \mu \mathrm{I}$ reaction system in the $0.2 \mathrm{ml}$ PCR tube. Each reaction contains $10 \mu \mathrm{I}$ of PrimeSTAR (TAKARA) PCR premix, $1 \mu$ I of forward primer (10nM) and $1 \mu$ I of reverse primer $(10 \mathrm{nM}), 10 \mathrm{ng}$ of sample DNA and $\mathrm{ddH}_{2} \mathrm{O}$ to supplement the volume to $20 \mu \mathrm{I}$. The PCR reactions were processed for 35 cycles on an Eppendorf thermocycler with denaturation at $94^{\circ} \mathrm{C}$ for 15 seconds, annealing at $58^{\circ} \mathrm{C}$ for 15 seconds, and extension at $72^{\circ} \mathrm{C}$ for 20 seconds. DNA electrophoresis was processed in 1\% agarose gel in TAE buffer. Primer sequences are KP-F: 5'TCT CAA TAA CAC CGA GCA-3', KP-R: 5'-TGC TCG GTG TTA TTG AGA-3'.

qPCR reactions were processed using Hieff UNICON Universal Blue qPCR SYBR Green Master Mix (Yeasen) on QuantStudio Dx (ABI). Programed started with a $95^{\circ} \mathrm{C}$ for 2 min followed by 40 cycles of denaturation $95^{\circ} \mathrm{C}$ for 10 seconds, annealing at $60^{\circ} \mathrm{C}$ for 10 seconds, and extension at $72^{\circ} \mathrm{C}$ for 15 seconds qPCR. Primers sequences are qKP-F: 5'-TCT CAA TAA CAC CGA GCA-3', qKP-R: 5'-TGC TCG GTG TTA TTG AGA-3'. Each reaction was repeated 3 biologically independent experiments. 
PCR-LbCas12a detection

The LbCas12a detection was carried in $20 \mu$ I system. The system contains 2 ul Buffer 3 (NEB),

50nM IbCas12a protein, 60nM of crRNA and 30nM labeled probe, and 100ng of purified PCR product.

Samples were mixed and then incubated at $37^{\circ} \mathrm{C}$, and signals were obtained from QuantStudio Dx

$(\mathrm{ABI})$ every minute for $60 \mathrm{~min}$. Each reaction was repeated 3 biologically independent experiments.

Study approval

The Luohu Ethnics Comittee approved the project (LLBGS [2021] 020). 


\section{Results}

\section{Establishment of PCR-IbCas12}

To find the suitable target for nucleic acid detection, we use blast to screen the most suitable gene as a target for nucleic acid detection. Results showed that IF-2, 16S RNA, and YP_005224572.1 are both specific and conservative genome fragments. Therefore, we use these three genes as candidates. By considering the TTTV PAM sequences, we designed five 36nt long crRNAs which share 19nt standards nucleotides and have 17nt unique sequences to base-pair $K$. pneumoniae targets. To quickly test the detecting efficiency of the crRNAs, synthetic singlestranded DNA targets were added to the IbCas12a system. Compared to the negative control, all crRNAs showed a significantly high level of FAM signals (Fig. 1A). Among the 5 crRNA sequences, crRNA-2 and crRNA-4 showed the most potent signals (Fig. 1B).

On the other hand, the primers to amplify DNA fragments for crRNA-2 and crRNA-4 are tested. The amplification efficiency of IF-2 primers is far more efficient than that of YP_005224572.1. As a result, crRNA-4 and the primers targeting the IF-2 gene are used to establish this system to detect the K. pneumoniae strain (Fig. 1C).

\section{LbCas12A showed superior sensitivity and specificity in K. pneumoniae detection}

First, to explore the minimum amount of DNA sample required for nucleic acid detection, serial diluted standard DNA samples were to test PCR, qPCR, and PCR-IbCas12a techniques. Basic PCR and qPCR exhibited positive signals when target DNA was as few as ten copies (Fig. 2A, B, C), 
while the LbCas12a system can exhibit signal in 40 min when the copy number is as few as one single copy (Fig. 2A). Next, to confirm if the detection system's specificity targets only $K$. pneumoniae, we applied IbCas $12 \mathrm{a}$ detection in 4 commonly seen bacteria strains such as Escherichia coli (E. coli), Staphylococcus aureus (S. aureus), Shigella Castellani, and Salmonella Typhimurium. As a result, K. pneumoniae exhibited as strong as 7 -folds of fluorescent signals in this system, while the rest bacteria strains did not (Fig. 2D). Thus, the results showed that the PCR-LbCas12a detection system is sensitive and specific.

\section{PCR-LbCas12 detecting Clinical samples}

We collected 21 positive and seven negative samples diagnosed and confirmed by plate culture examination from the laboratory department to apply this system to clinical use. 21 positive $K$. pneumoniae were also positive in the PCR-LbCas12a detection system (Fig. 3A, B, C). However, one out of the seven negative was tested positive. Basic PCR was processed to confirm this case, and its product was sent for sanger sequencing. Sequencing fully matched the K. pneumoniae conservative IF-2 sequence, which means that the sample was indeed positive. A similar Cas $12 a$ detecting system named DETECTR used RPA instead of PCR to amplify target DNA to save the thermo-cycling process, which is more appliable to clinical use (Kostyusheva et al. 2021). We also tried this system to detect the same samples. However, compared to our system, their signals were far less significant than satisfactory (Fig. S1A, B). 


\section{Discussion}

Recent studies revealed that about $73.1 \%$ of $K$. pneumoniae are resistant to at least one antibiotic [15]. The rate of colistin resistant strains is still increasing [16]. The infection of $K$. pneumoniae could cause untreatable diseases that could threaten patients' lives. Therefore, the detection of $K$. pneumoniae from patients and ventilators is vital to prevent infections.

The most used detection method is agar well diffusion assay culture [17]. However, the sensitivity and accuracy are only about $56 \%$ and $65 \%$ [18]. As a comparison, the detection of nucleic acid is more advantageous in stability and accuracy. The golden standard of nucleic acid detection is qPCR. qPCR specifically and accurately identifies K. pneumoniae from bacteria like E. coli and S. aureus [19] and its antibiotic-resistant genes, such as ESBL-encoding and Fosfomycin-encoding genes [20]. However, qPCR needs expensive equipment and skilled workers[21]. Recently, CRISPR-Cas12 mediated trans-collateral activity was widely applied to nucleic acid detection $[11,22,23]$. The CRISPR-Cas12 detection system is accurate and specific, and it can also combine various readout and amplification technologies [24-26].

In this study, we first established the LbCas12a system to detect K. pneumoniae nucleic acid. Before the Cas12 reaction, we used PCR to harvest enough amount of target DNA as substrate. Although the RPA technology can also accomplish this process, however, according to our experiment, PCR is more stable and cheaper (Fig. S1A, B). As crRNA could be as short as 36nt, we used chemically synthesized crRNA instead of in vitro. transcribed RNA. After selection and finetuning of primers and crRNAs, the PCR-LbCas12a detection system can detect as low as only one copy of K. pneumoniae. We also tried the system in samples to explore its possibility in clinical use. Samples were clinically diagnosed by Agar Well Diffusion Assay. After PCR-LbCas12a detection, 
this system successfully checked out all positive samples. To our surprise, one of the negative samples turned out to be a positive case. Additionally, considering the time cost in Agar Well Diffusion Assay culture, which requires 24-48 hours, the PCR-IbCas12a system needs no more than 4 hours. Therefore, this detecting system is a better option in most cases.

The experiment supports that the PCR-LbCas12a system targeting K. pneumoniae is a sensitive, accurate and easy-to-use technology to detect $K$. pneumoniae nucleic acid.

\section{FUNDING}

This study was supported by the National Natural Science Foundation of China (81922046, 61931024, and 81802741), the Special Funds for Strategic Emerging Industries Development in Shenzhen (20180309163446298), Shenzhen Science and Technology Innovation Commission (RCJC20200714114557005) and Shenzhen Key Laboratory Program (ZDSYS20190902092857146). The China Postdoctoral Science Foundation (2020M682949). The Guangdong Basic and Applied Basic Research Foundation (2020A1515110908).

\section{COMPETING INTERESTS}

The authors declare that they have no competing interests. 


\section{REFERENCES}

1. Magill, S.S.; Edwards, J.R.; Bamberg, W.; Beldavs, Z.G.; Dumyati, G.; Kainer, M.A.; Lynfield, R.; Maloney, M.; McAllister-Hollod, L.; Nadle, J.; et al. Multistate point-prevalence survey of health care-associated infections. N Engl J Med 2014, 370, 1198-1208, doi:10.1056/NEJMoa1306801.

2. Podschun, R.; Ullmann, U. Klebsiella spp. as nosocomial pathogens: epidemiology, taxonomy, typing methods, and pathogenicity factors. Clin Microbiol Rev 1998, 11, 589-603, doi:10.1128/CMR.11.4.589.

3. Haghighifar, E.; Norouzi, F.; Kamali Dolatabadi, R. Molecular detection of Extended-Spectrum beta-lactamases (ESBLs) and biofilm formation in uropathogen Klebsiella pneumoniae in Iran. Med J Islam Repub Iran 2021, 35, 72, doi:10.47176/mjiri.35.72.

4. Wagner, S.J.; Benjamin, R.J.; Hapip, C.A.; Kaelber, N.S.; Turgeon, A.M.; Skripchenko, A.; Stassinopoulos, A. Investigation of bacterial inactivation in apheresis platelets with 24 or 30 hours between inoculation and inactivation. Vox Sang 2016, 111, 226-234, doi:10.1111/vox.12410.

5. Hutchison, J.R.; Piepel, G.F.; Amidan, B.G.; Hess, B.M.; Sydor, M.A.; Deatherage Kaiser, B.L. Comparison of false-negative rates and limits of detection following macrofoam-swab sampling of Bacillus anthracis surrogates via Rapid Viability PCR and plate culture. J Appl Microbiol 2018, 124, 1092-1106, doi:10.1111/jam.13706.

6. Johnson, G.; Millar, M.R.; Matthews, S.; Skyrme, M.; Marsh, P.; Barringer, E.; O'Hara, S.; Wilks, M. Evaluation of BacLite Rapid MRSA, a rapid culture based screening test for the detection of ciprofloxacin and methicillin resistant S. aureus (MRSA) from screening swabs. BMC Microbiol 2006, 6, 83, doi:10.1186/1471-2180-6-83.

7. Hyun, M.; Lee, J.Y.; Ryu, S.Y.; Ryoo, N.; Kim, H.A. Antibiotic Resistance and Clinical Presentation of Health Care-Associated Hypervirulent Klebsiella pneumoniae Infection in Korea. Microb Drug Resist 2019, 25, 1204-1209, doi:10.1089/mdr.2018.0423.

8. Yan, W.X.; Hunnewell, P.; Alfonse, L.E.; Carte, J.M.; Keston-Smith, E.; Sothiselvam, S.; Garrity, A.J.; Chong, S.; Makarova, K.S.; Koonin, E.V.; et al. Functionally diverse type V CRISPR-Cas systems. Science 2019, 363, 88-91, doi:10.1126/science.aav7271.

9. Chen, J.S.; Ma, E.; Harrington, L.B.; Da Costa, M.; Tian, X.; Palefsky, J.M.; Doudna, J.A. CRISPRCas12a target binding unleashes indiscriminate single-stranded DNase activity. Science 2018, 360, 436-439, doi:10.1126/science.aar6245.

10. Gootenberg, J.S.; Abudayyeh, O.O.; Kellner, M.J.; Joung, J.; Collins, J.J.; Zhang, F. Multiplexed and portable nucleic acid detection platform with Cas13, Cas12a, and Csm6. Science 2018, 360, 439-444, doi:10.1126/science.aaq0179.

11. Li, L.; Li, S.; Wu, N.; Wu, J.; Wang, G.; Zhao, G.; Wang, J. HOLMESv2: A CRISPR-Cas12b-Assisted Platform for Nucleic Acid Detection and DNA Methylation Quantitation. ACS Synth Biol 2019, 8, 2228-2237, doi:10.1021/acssynbio.9b00209.

12. Kostyusheva, A.; Brezgin, S.; Babin, Y.; Vasilyeva, I.; Glebe, D.; Kostyushev, D.; Chulanov, V. CRISPR-Cas systems for diagnosing infectious diseases. Methods 2021, doi:10.1016/j.ymeth.2021.04.007.

13. Nouri, R.; Tang, Z.; Dong, M.; Liu, T.; Kshirsagar, A.; Guan, W. CRISPR-based detection of SARSCoV-2: A review from sample to result. Biosens Bioelectron 2021, 178, 113012, doi:10.1016/j.bios.2021.113012. 
14. Li, S.Y.; Cheng, Q.X.; Liu, J.K.; Nie, X.Q.; Zhao, G.P.; Wang, J. CRISPR-Cas12a has both cis- and trans-cleavage activities on single-stranded DNA. Cell Res 2018, 28, 491-493, doi:10.1038/s41422-018-0022-x.

15. Sharahi, J.Y.; Hashemi, A.; Ardebili, A.; Davoudabadi, S. Molecular characteristics of antibioticresistant Escherichia coli and Klebsiella pneumoniae strains isolated from hospitalized patients in Tehran, Iran. Ann Clin Microbiol Antimicrob 2021, 20, 32, doi:10.1186/s12941-021-00437-8.

16. Petrosillo, N.; Taglietti, F.; Granata, G. Treatment Options for Colistin Resistant Klebsiella pneumoniae: Present and Future. J Clin Med 2019, 8, doi:10.3390/jcm8070934.

17. Prastiyanto, M.E.; Tama, P.D.; Ananda, N.; Wilson, W.; Mukaromah, A.H. Antibacterial Potential of Jatropha sp. Latex against Multidrug-Resistant Bacteria. Int J Microbio/ 2020, 2020, 8509650, doi:10.1155/2020/8509650.

18. Koyuncu, S.; Haggblom, P. A comparative study of cultural methods for the detection of Salmonella in feed and feed ingredients. BMC Vet Res 2009, 5, 6, doi:10.1186/1746-6148-5-6.

19. Kim, S.H.; Lee, J.S.; Lee, J.H.; Kim, Y.J.; Choi, J.G.; Lee, S.K.; Kim, H.J.; Yang, S.J.; Park, T.; Lee, S.K.; et al. Development and Application of a Multiplex Real-Time Polymerase Chain Reaction Assay for the Simultaneous Detection of Bacterial Aetiologic Agents Associated With Equine Venereal Diseases. J Equine Vet Sci 2021, 105, 103721, doi:10.1016/j.jevs.2021.103721.

20. Castanheira, M.; Johnson, M.G.; Yu, B.; Huntington, J.A.; Carmelitano, P.; Bruno, C.; Rhee, E.G.; Motyl, M. Molecular Characterization of Baseline Enterobacterales and Pseudomonas aeruginosa Isolates from a Phase 3 Nosocomial Pneumonia (ASPECT-NP) Clinical Trial. Antimicrob Agents Chemother 2021, 65, doi:10.1128/AAC.02461-20.

21. Corman, V.M.; Landt, O.; Kaiser, M.; Molenkamp, R.; Meijer, A.; Chu, D.K.; Bleicker, T.; Brunink, S.; Schneider, J.; Schmidt, M.L.; et al. Detection of 2019 novel coronavirus (2019-nCoV) by realtime RT-PCR. Euro Surveill 2020, 25, doi:10.2807/1560-7917.ES.2020.25.3.2000045.

22. Selvam, K.; Najib, M.A.; Khalid, M.F.; Mohamad, S.; Palaz, F.; Ozsoz, M.; Aziah, I. RT-LAMP CRISPR-Cas12/13-Based SARS-CoV-2 Detection Methods. Diagnostics (Basel) 2021, 11, doi:10.3390/diagnostics11091646.

23. Ma, L.; Peng, L.; Yin, L.; Liu, G.; Man, S. CRISPR-Cas12a-Powered Dual-Mode Biosensor for Ultrasensitive and Cross-validating Detection of Pathogenic Bacteria. ACS Sens 2021, 6, 29202927, doi:10.1021/acssensors.1c00686.

24. Ali, Z.; Aman, R.; Mahas, A.; Rao, G.S.; Tehseen, M.; Marsic, T.; Salunke, R.; Subudhi, A.K.; Hala, S.M.; Hamdan, S.M.; et al. iSCAN: An RT-LAMP-coupled CRISPR-Cas12 module for rapid, sensitive detection of SARS-CoV-2. Virus Res 2020, 288, 198129, doi:10.1016/j.virusres.2020.198129.

25. Chen, Y.; Shi, Y.; Chen, Y.; Yang, Z.; Wu, H.; Zhou, Z.; Li, J.; Ping, J.; He, L.; Shen, H.; et al. Contamination-free visual detection of SARS-CoV-2 with CRISPR/Cas12a: A promising method in the point-of-care detection. Biosens Bioelectron 2020, 169, 112642, doi:10.1016/j.bios.2020.112642.

26. Ramachandran, A.; Huyke, D.A.; Sharma, E.; Sahoo, M.K.; Huang, C.; Banaei, N.; Pinsky, B.A.; Santiago, J.G. Electric field-driven microfluidics for rapid CRISPR-based diagnostics and its application to detection of SARS-CoV-2. Proc Natl Acad Sci U S A 2020, 117, 29518-29525, doi:10.1073/pnas.2010254117. 


\section{Figures}

\section{Figure.1}

A

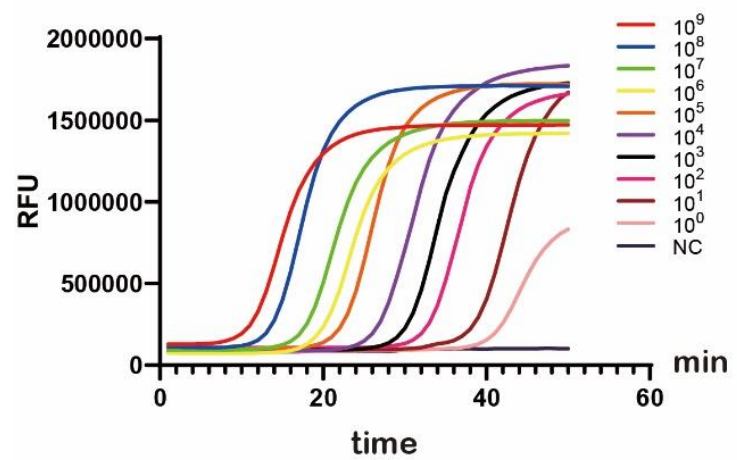

C

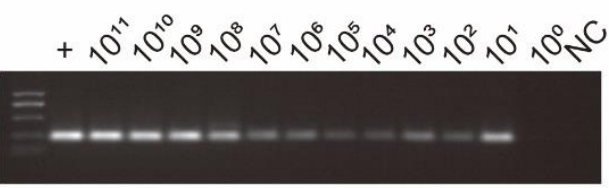

B

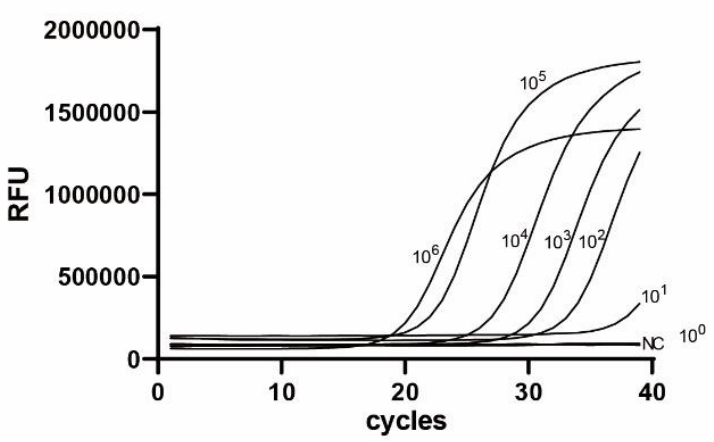

D

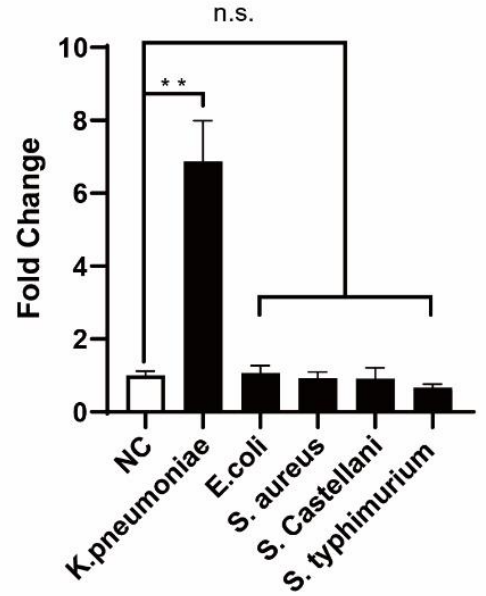




\section{Figure 2}

A

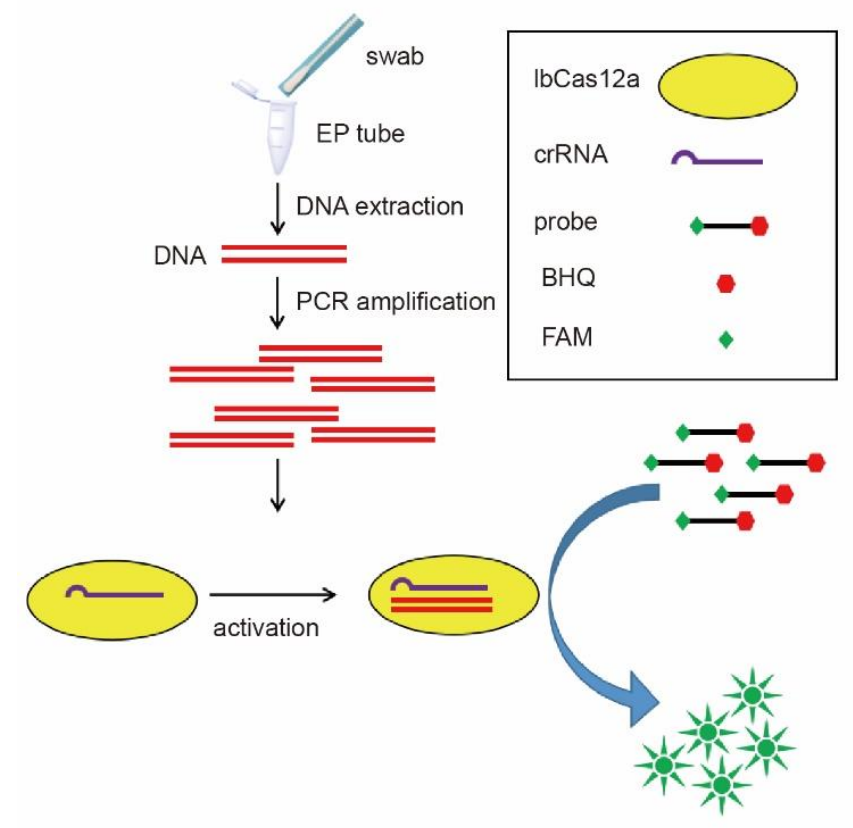

B
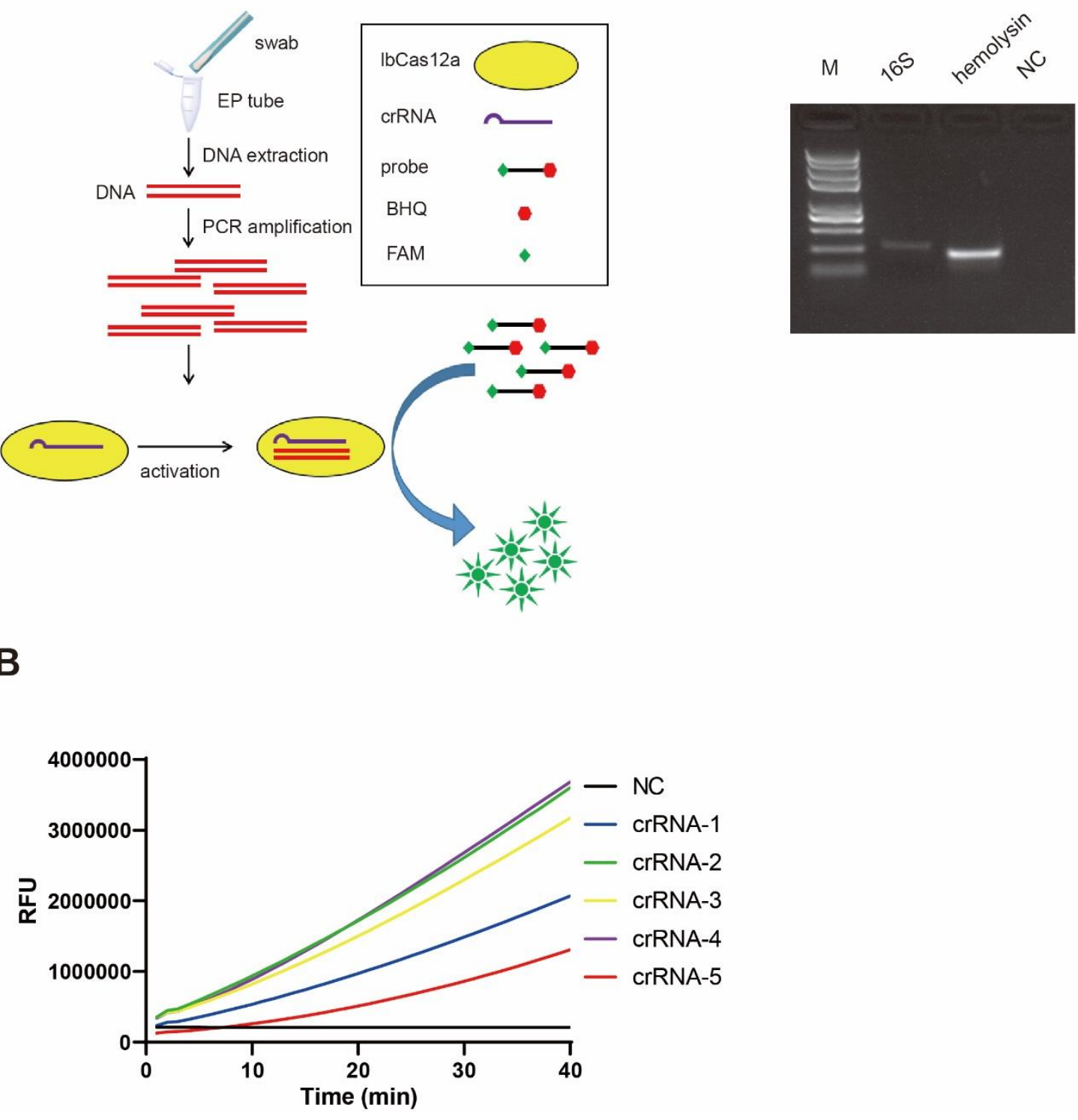

C 


\section{Figure3}

A

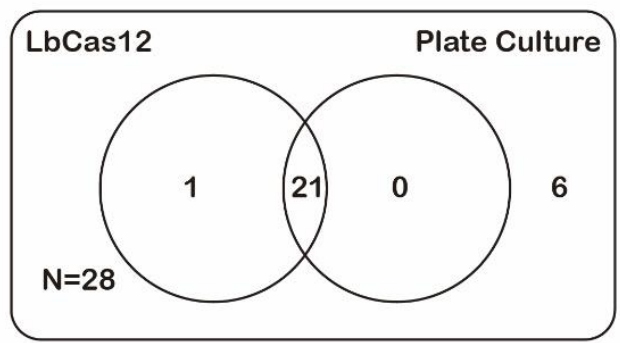

Clinical Samples

C

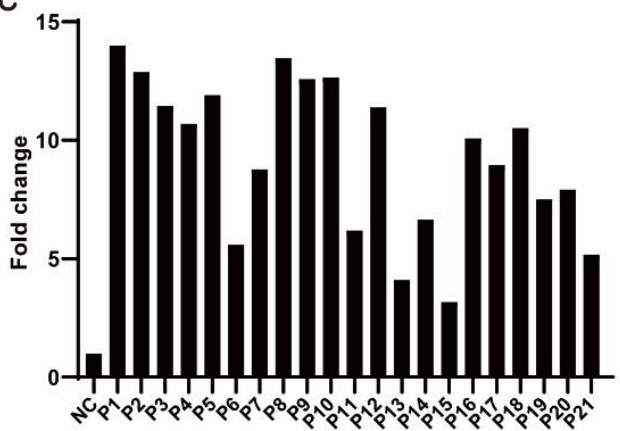

B

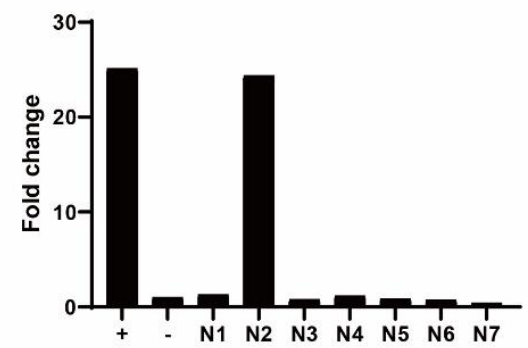

D

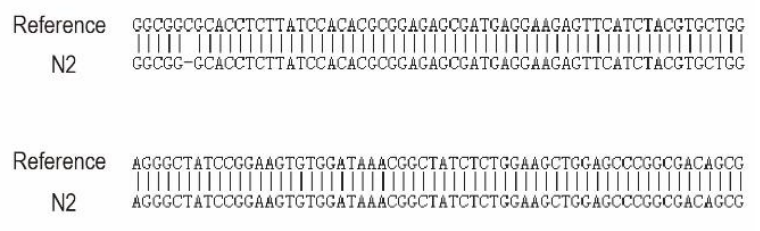




\section{FIGURES LEGENDS}

Figure 1. A. Graphical illustration of LbCas12a detecting K. pneumoniae nucleic acid. B. Tests of 5 crRNA efficiency in LbCas12a detecting system. C. Electrophoresis of PCR product of K. pneumoniae 16S and YP_005224572.1 gene.

Figure 2. A. The RFU signal generated by the PCR-LbCas12 system detecting the serially diluted standard $K$. pneumoniae DNA samples. B. The qPCR results of serial diluted standard DNA samples. C. $1 \%$ agarose gel electrophoresis of the PCR product from serial diluted standard DNA samples. D. Different bacterium was detected by PCR-Cas12 system. Only K. pneumoniae was successfully detected. Data are mean $\pm s$. $d$. of $n=3$ biological independent experiments.

Figure 3. A. Venn diagrams comparing PCR-LbCas12 system and plate culture in clinically diagnosed samples. B. Seven clinical negative samples were tested in the PCR-LbCas12 system, but N2 is positive. C. 21 positive samples were all tested positive in the PCR-LbCas12 system. D. Sanger sequencing result of $\mathrm{N} 2$ was highly consistent with standard $K$. pneumoniae sequences. 


\section{Supplementary information}

Figure S1. A. seven negative clinical samples were tested in the RPA-LbCas12 system. B. 21 positive samples were all tested positive in the RPA-LbCas12 system. 